described. Dr J. F. W. Bell (University of Aston) has used the variation of the velocity of sound with temperature in a solid as the principle behind his thermometer. Mr E. S. Morgan (Central Electricity Research Laboratories, Leatherhead), on the other hand, used this variation in a gas. Morgan used his instrument to measure the average temperature of the gases in a furnace by measuring the time taken for a pulse of sound to travel across the furnace.

\section{VITREOUS STATE}

\section{Atoms and Glasses}

\section{from a Correspondent}

Some busy experts on the vitreous state managed to fit a Faraday Society discussion on this topic into their conference schedule between September 22 and 24 . About half of the contributions presented at Bristol were devoted to electrons or phonons in vitreous materials, and the rest were largely concerned with nucleation, crystal growth and phase separation in glass forming systems. Introducing the electron and phonon section, Professor Sir Nevill Mott (University of Cambridge) reviewed the state of knowledge of conduction mechanisms in amorphous and liquid semiconductors. The existence of localized electron states in these materials has been accepted for some time, and the chief contention centres on the existence and position of the so-called mobility edge, which may divide the electron energy band into localized and nonlocalized regions. The effectiveness of electron-electron interactions in modifying the already quite complex theoretical picture has also been the subject of much recent discussion. It was taken up again by Professor M. Pollak (University of California) whose chief conclusion was that the interaction can significantly affect activation energies associated with d.c. conductivity in various ways, as well as increasing the a.c. conductivity above a certain critical frequency.

Mr T. Klima and Mr T. MeGill (University of Bristol) raised some comment when they dealt with the role of short range order in producing an energy gap in covalent amorphous semiconductors. But, on the whole, phonons sparked off much more discussion than did electrons. Significant theoretical progress is being made on several fronts, for example, with the model based calculations of Bell and Dean, the crystalline approach of Gaskell and the orientationally disordered molecular crystal methods developed by Whalley and his colleagues.

The second half of the discussion was prefaced by Professor J. Zarzycki (Saint-Gobain, Paris) who reviewed phase separated systems. He touched on the failure of some stoichiometric and chemical composition based theories to account for the temperature dependence of miscibility phenomena. He also outlined the basis of the spinodal analysis and remarked on the difficulty in distinguishing between nucleation and spinodal growth mechanisms by small angle X-ray scattering methods; the discussion returned to this difficulty several times.

The significance of Cahn's spinodal analysis was called into question several times, with some particularly pointed evidence for the prosecution coming from Dr R. K. MacCrone (Rensselaer Institute, New York). It is perhaps natural that theories in this area of amorphous research should be inherently more macro- scopic or coarse grained in character than those in the realm of electrons or phonons. But there seems to be a growing prospect that the molecular dynamies, or computer simulation techniques already exploited in connexion with simple liquids, can make possible significant progress during the next decade in probing nucleation and miscibility phenomena at the atomic level.

\section{MICROSCOPY}

\section{More Scope for Scunning}

\section{from our Materials Science Correspondent}

From a rare laboratory curiosity, the scanning electron microscope has in five years developed into a tool widely used in materials science, microelectronics, biology and even the assessment of lunar dust. Most routine users exploit the instrument in one mode only - the emissive mode, which is used to give information about surface topography. They will be helped to make more varied use of their instruments by $D$. R. Clarke's recent survey of image contrast in the SEM (J. Mat. Sci., 5, 689; 1970).

Beginning with the standard emissive mode of image contrast, Clarke distinguishes clearly between backscattered and secondary electrons and details the special contributions to contrast made by each of these electron types. An important innovation is MacDonald's use of Auger electrons to form an image: this allows highly sensitive atomic number contrast (which is otherwise rather poor in the SEM), and also allows voltage contrast-used for examining microcircuits in operationto be sharply separated from other forms of contrast. Topographic contrast is interpreted in some detail and the roles of secondary and back-scattered electrons are distinguished.

After a survey of voltage and electric field contrast, of magnetic domain contrast and of beam-induced conductivity contrast, which is used to reveal defects in semiconductors, Clarke surveys very recent developments in distinguishing different crystal orientations. This aspect of image contrast depends on the formation of channelling patterns (a term borrowed from ionimplantation) and indeed the orientation of a crystal can often be determined, though the modest magnification which can be used in this connexion remains a limitation.

Clarke goes on to detail improvements in the collector and display systems (though not referring to the improvement of atomic number contrast which can be achieved by using twin collectors-a Japanese contribution). Electronic processing developed at the Services Electronic Research Laboratory, Baldock, has macle it possible for contoured and "quantized" displays to replace simple brightness modulations in images, and a form of stroboscopic display can be used to slow down and display rapid periodic voltage sequences in a microelectronic device.

\section{STRESS ANALYSIS}

\section{Computers to the Fore}

\section{from a Correspondent}

STRESS analysis is one of many enginecring subjects which are becoming dominated by digital computer techniques. In this case the technique is finite element 